\title{
PAPR for OFDM and the Proportion of Information Bearing Signals for Tone Reservation
}

\author{
Holger Boche, Brendan Farrell \\ Heinrich-Hertz-Lehrstuhl für Informationstheorie und Theoretische Informationstechnik \\ Technische Universität Berlin \\ Einsteinufer 25, 10587 Berlin, Germany \\ Email: holger.boche@mk.tu-berlin.de,farrell@mk.tu-berlin.de
}

\begin{abstract}
We consider the performance of tone reservation for reduction of the Peak-to-Average Power Ratio (PAPR) in OFDM signals. Tone reservation is unique among methods for reducing PAPR because it does not affect information bearing coefficients and involves no additional coordination of transmitter and receiver. It is shown that if the OFDM system always satisfies a given peak-to-average power ratio constraint, then the efficiency of the system, defined as the ratio of the number of tones used for information to the entire number of tones used, must converge to zero as the total number of tones increases.
\end{abstract}

\section{INTRODUCTION}

Orthogonal Frequency Division Multiplexing (OFDM) is one of today's most widely used and promising information transmission schemes. One of the main disadvantages of OFDM, however, is large Peak-to-Average Power Ratios (PAPR) of the transmit signals. Reducing the PAPR, which we will call the PAPR reduction problem, has been an area of extensive research over the last ten years, and various techniques have been proposed. These include, among others, clipping and filtering, selected mapping, active constellation extension, and tone reservation. See [1] for an overview.

All of these schemes begin with a set of coefficients to be transmitted to the receiver. One may adjust these coefficients in some way or add new coefficients on frequencies that have not been used. If coefficients are manipulated, then the receiver must convert the received coefficients back to the original coefficients; however, if coefficients are added on frequencies that do not carry information, the received information bearing coefficients do not have to be converted. Tone reservation, which was introduced in [2], and is one of the popular techniques to mitigate against high PAPR, takes the latter approach.

In this scheme, the set of available tones is divided into two subsets. One set is used to carry information, while the other is used to reduce the peak value. We will call these two sets the information set and the compensation set. Given a set of coefficients for the tones in the information set, coefficients are chosen for tones in the compensation set, so that the peak value of the combined signal is reduced. the location of these

This work was supported by the German Science Foundation (DFG) under Project BO 1734/18-1 two sets remain fixed for all codewords and over all uses of the channel.

Of the handful of methods to reduce PAPR, tone reservation is particularly robust and canonical. This is because the only information that the receiver requires is the location of the information set. The receiver may simply ignore whatever arrives on the entries of the compensation set. With other schemes, such as active constellation extension or selected mapping, not only does the receiver have to be informed of the modifications made to each possible set of coefficients, but the receiver also has to convert the coefficients back to their original values. Thus there is additional overhead involved in establishing setting up and then performing the information transmission. Both of these are avoided in tone reservation.

However, tone reservation exhibits a trade-off between the best attainable PAPR and the number of tones in the information set. The main result presented here is: if the OFDM system satisfies a strict bound on the peak to average ratio, then as the number of total tones used increases, at some point the proportion of tones used to carry information must decrease and eventually converge to zero. Equivalently, we find a scaling law: if the size of the information set and the total set increase proportionally, signals with larger peaks can be constructed that cannot be compensated for by any compensation signal.

The result presented here certainly does not state that tone reservation does not deliver strong improvements in PAPR. An efficient algorithm for computing compensation coefficients is given in [3], and the reductions it delivers in PAPR are significant. Much experimentation has been done, in particular in searching for subsets with good performance, but the structure of good sets is still not understood. However, there has been little theoretical work on the performance bounds of tone reservation. The authors are unaware, for example, of any work that addresses the behavior of tone reservation as the number of tones increases. This paper provides insight to the scheme as the number of tones involved becomes large.

In Section II we prove our main result on tone reservation for finite-dimensional OFDM systems. In Section III we prove the same type of result for infinite-dimensional systems. Finally, in Section IV we discuss the analogous questions in the discrete setting. 


\section{The Finite Set OFDM CASE}

We first define our signals: an OFDM signal has the form

$$
s(t)=\sum_{k=-N}^{N} a_{k} e^{i k t},
$$

where the coefficients $a_{k}$ either carry information or, in the tone reservation scheme, are used to reduce the peak value of $s(t)$. The PAPR is

$$
\operatorname{PAPR}(a)=\sup _{t \in[0,2 \pi]} \frac{\left|\sum_{k=-N}^{N} a_{k} e^{i k t}\right|}{\|a\|_{l_{2 N+1}^{2}}} .
$$

When using tone reservation, we split $\{-N, \ldots, N\}$ into two subsets $I_{N}$, an information set, and $R_{N}=\{-N, \ldots, N\} \backslash I_{N}$, a compensation set. We call the ratio of the number of tones in the information set to the total number of tones available the efficiency of the system. Using $|A|$ to denote the number of elements in the set $A$, the efficiency is $\left|I_{N}\right| /(2 N+1)$.

Given a set of information coefficients, one then seeks to choose coefficients $b$ supported on the compensation set to reduce the peak of the combined signal. Before we formally state the problem we define our spaces.

Definition 2.1: $l_{N}^{p}$ denotes $\mathbb{C}^{N}$ viewed as a linear space with the norm

$$
\|x\|_{l_{N}^{p}}=\left(\sum_{k=1}^{N}\left|x_{k}\right|^{p}\right)^{1 / p} .
$$

If $A$ is a subset of $\{-N, \ldots, N\}, l^{p}(A)$ denotes vectors $l_{2 N+1}^{p}$ supported on $A . L^{p}(\mathbb{T})$ denotes $p$-integrable functions defined on $\mathbb{T}$ with norm

$$
\|f\|_{L^{p}(\mathbb{T})}=\left(\frac{1}{2 \pi} \int_{\mathbb{T}}|f(t)|^{p} d t\right)^{1 / p}
$$

for $1 \leq p<\infty$ and

$$
\|f\|_{L^{\infty}(\mathbb{T})}=\operatorname{ess.sup}_{t \in \mathbb{T}}|f(t)| .
$$

$L^{2}\left(I_{N}\right)$ denotes the subspace of $L^{2}(\mathbb{T})$ spanned by $\left\{e^{i k \cdot}\right\}_{k \in I_{N}}$.

Tone reservation works as follows: given a subset $I_{N}$ of $\{-N, \ldots, N\}$, and a vector $a \in l^{2}\left(I_{N}\right)$, we seek a vector $b \in l_{2 N+1}^{2}$ supported on $R_{N}$ and satisfying $\|b\|_{l_{2 N+1}^{2}} \leq$ $C_{E x}\|a\|_{l_{2 N+1}^{2}}$, such that

$$
\sup _{t \in[0,2 \pi]}\left|\sum_{k \in I_{N}} a_{k} e^{i k t}+\sum_{k \in R_{N}} b_{k} e^{i k t}\right| \leq C_{\mathrm{Ex}}\|a\|_{l_{2 N+1}^{2}},
$$

for some constant $C_{\mathrm{Ex}}$.

The condition $\|b\|_{l_{2 N+1}^{2}} \leq C_{\mathrm{Ex}}\|a\|_{l_{2 N+1}^{2}}$ is made so that the infimum over all possible $b$ supported on $R_{N}$ is well-defined. We note, though, that any vector $b$ that satisfies (6) must have this property. To see this, we observe that

$$
\begin{aligned}
\left(\|a\|_{l^{2}}^{2}+\|b\|_{l^{2}}^{2}\right)^{1 / 2} & =\left\|\sum_{k \in I_{N}} a_{k} e^{i k \cdot}+\sum_{k \in R_{N}} b_{k} e^{i k \cdot}\right\|_{L^{2}(\mathbb{T})} \\
\leq & \sup _{t \in[0,2 \pi]}\left|\sum_{k \in I_{N}} a_{k} e^{i k t}+\sum_{k \in R_{N}} b_{k} e^{i k t}\right|,
\end{aligned}
$$

so that if inequality (6) holds, then the condition $\|b\|_{l_{2 N+1}^{2}} \leq$ $C_{\text {Ex }}\|a\|_{l_{2 N+1}^{2}}$ is also satisfied. We comment that for finite $N$, a constant $C$ can always be found that satisfies inequality (6). Here we address the relationship between $N$, the size of $I_{N}$ and the best possible constant $C_{\mathrm{Ex}}$.

Determining the signal with reduced peak value given the vector $a$ can be viewed as a nonlinear operator, which we will call the extension operator and denote $E_{I_{N}}$. This operator is a map from $l^{2}\left(I_{N}\right)$ to $L^{2}(\{-N, \ldots, N\})$, given by

$$
E_{I_{N}} a=\sum_{k \in I_{N}} a_{k} e^{i k t}+\sum_{k \in R_{N}} b_{k} e^{i k t} .
$$

An initial formulation of solvability is: the PAPR reduction problem is solvable for the subset $I_{N} \subset\{-N, \ldots, N\}$ with bound $C_{\mathrm{Ex}}$ if there exists an operator $E_{I_{N}}: l^{2}\left(I_{N}\right) \rightarrow$ $L^{\infty}(\{-N, \ldots, N\})$ such that for all $a \in l^{2}\left(I_{N}\right)$ satisfying $\|a\|_{l_{2 N+1}^{2}} \leq 1$

$$
\left\|E_{I_{N}} a\right\|_{L^{\infty}(\mathbb{T})} \leq C_{\mathrm{Ex}} .
$$

Such an operator will, in general, be non-linear since cases where $E_{I_{N}}(a+b) \neq E_{I_{N}} a+E_{I_{N}} b$ will exist. However, the operator scales sub-linearly. That is, suppose that the PAPR reduction problem as just defined is solvable for $I_{N}$ with constant $C_{\mathrm{Ex}}$. If $\|a\|_{l_{2 N+1}^{2}}>1$, we define $a^{\prime}=\frac{a}{\|a\|_{l_{2 N+1}^{2}}}$. Then

$$
\left\|E_{I_{N}} a^{\prime}\right\|_{L^{\infty}(\mathbb{T})} \leq C_{\mathrm{Ex}},
$$

and we may simply rescale $E_{I_{N}} a^{\prime}$ by $\|a\|_{l_{2 N+1}^{2}}$ to determine an extension for $a$ with bound $C_{\mathrm{Ex}}\|a\|_{l_{2 N+1}^{2}}$. Thus, solvability on all of $l^{2}\left(I_{N}\right)$ and solvability on the unit ball of $l^{2}\left(I_{N}\right)$ are equivalent, though the best constant in the latter case may be smaller.

Definition 2.2: The PAPR reduction problem is solvable for $I_{N}$ with bound $C_{\mathrm{Ex}}$ if there exists an operator $E_{I_{N}}: l^{2}\left(I_{N}\right) \rightarrow$ $L^{\infty}(\{-N, \ldots, N\})$ such that

$$
\left\|E_{I_{N}} a\right\|_{L^{\infty}(\mathbb{T})} \leq C_{\mathrm{Ex}}\|a\|_{l_{2 N+1}^{2}}
$$

for every $a \in l^{2}\left(I_{N}\right)$.

Now we proceed as follows: in Theorem 2.4 we give a necessary condition for solvability. With Theorem 2.6 we show that if it is required that the peak-to-average power ratio remains bounded, then the efficiency of the OFDM system converges to zero as the system size increases. Equivalently, if the PAPR reduction problem remains solvable for a sequence of sets $\left\{I_{N}\right\}$ as $N \rightarrow \infty$, then the efficiency of the sets must converge to zero.

Definition 2.3: For a subset $I_{N} \subset\{-N, \ldots, N\}$ we define

$$
\mathcal{F}\left(I_{N}\right)=\left\{f \in L^{1}(\mathbb{T}), f(t)=\sum_{k \in I_{N}} a_{k} e^{i k t}\right\} .
$$

Theorem 2.4: If the PAPR problem is solvable for the subset $I_{N}$ with extension norm $C_{\mathrm{Ex}}$ then

$$
\|f\|_{L^{2}(\mathbb{T})} \leq C_{\mathrm{Ex}}\|f\|_{L^{1}(\mathbb{T})}
$$

for all $f \in \mathcal{F}\left(I_{N}\right)$. 
Proof: By assumption, for all $s(t)=\sum_{k \in I_{N}} a_{k} e^{i k t}$, $\|a\|_{l_{2 N+1}^{2}} \leq 1$,

$$
\left\|E_{I_{N}} a\right\|_{l_{2 N+1}^{\infty}} \leq C_{\mathrm{Ex}}\|a\|_{l_{2 N+1}^{2}} \leq C_{\mathrm{Ex}} .
$$

Again by assumption,

$$
\left(E_{I_{N}} a\right)(t)=\sum_{k \in I_{N}} a_{k} e^{i k t}+\sum_{k \in R_{N}} b_{k} e^{i k t} .
$$

Let $f \in \mathcal{F}\left(I_{N}\right), f(t)=\sum_{k \in I_{N}} c_{k} e^{i k t}$, be arbitrary. Then

$$
\begin{aligned}
\left|\sum_{k \in I_{N}} a_{k} \bar{c}_{k}\right| & =\left|\sum_{k \in I_{N}} a_{k} \bar{c}_{k}+\sum_{k \in R_{N}} b_{k} \bar{c}_{k}\right| \\
& =\left|\frac{1}{2 \pi} \int_{\mathbb{T}} f(t) \overline{\left(E_{I_{N}} a\right)(t)} d t\right| \\
& \leq\|f\|_{L^{1}(\mathbb{T})}\left\|E_{I_{N}} a\right\|_{L^{\infty}(T)} \\
& \leq\|f\|_{L^{1}(\mathbb{T})} C_{\mathrm{Ex}}\|a\|_{l_{2 N+1}^{2}} \\
& \leq C_{\mathrm{Ex}}\|f\|_{L^{1}(\mathbb{T})} .
\end{aligned}
$$

Set

$$
a_{k}=\left\{\begin{array}{cc}
\frac{c_{k}}{\|c\|_{l_{2 N+1}^{2}}} & c_{k} \neq 0 \\
0 & c_{k}=0
\end{array} .\right.
$$

Then

$$
\left\|\sum_{k \in I_{N}} a_{k} \bar{c}_{k} \mid=\right\| c\left\|_{l_{2 N+1}^{2}}=\right\| f\left\|_{L^{2}(\mathbb{T})} \leq C_{\mathrm{Ex}}\right\| f \|_{L^{1}(\mathbb{T})} .
$$

The following definition gives the efficiency of the best subset selection for which the PAPR reduction problem is solvable for a given bound.

Definition 2.5 (Optimal subset size):

$\mathcal{E}_{N}\left(C_{\mathrm{Ex}}\right)=\max \left\{\left|I_{N}\right| ; I_{N} \subset\{-N, \ldots, N\}\right.$, such that

PAPR is solvable for $I_{N}$ with constant $\left.C_{\mathrm{Ex}}\right\}$.

Now we may state the following theorem.

Theorem 2.6: For all $0<C_{\mathrm{Ex}}<\infty$, the following limit holds:

$$
\limsup _{N \rightarrow \infty} \frac{\mathcal{E}_{N}\left(C_{\mathrm{Ex}}\right)}{2 N+1}=0
$$

In words, the theorem states that if a PAPR bound is always satisfied, then the system efficiency converges to 0 as the total size increases. Thus the number of tones that may be used to carry information does not scale with $N$.

The proof will use arithmetic progressions and Szemerédi's Theorem.

Definition 2.7: An arithmetic progression of length $k$ is a subset of $\mathbb{Z}$ that has the form $\{a, a+d, a+2 d, \ldots ., a+(k-1) d\}$ for some integer $a$ and some positive integer $d$.

Theorem 2.8: (Theorem 1.2 in [4]) For any integer $k \geq 1$ and any $0<\delta \leq 1$, there exists an integer $N_{S Z}(k, \delta) \geq 1$ such that for every $N \geq N_{S Z}(k, \delta)$, every set $A \subset\{1, \ldots, N\}$ of cardinality $|A| \geq \delta N$ contains at least one arithmetic progression of length $k$.
Proof of Theorem 2.6: Assume that the claim is not true. Then there exists a subsequence $\left\{N_{k}\right\}_{k=1}^{\infty} \subset \mathbb{N}$ and a constant $G\left(C_{\mathrm{Ex}}\right)$ such that

$$
\frac{\mathcal{E}_{N_{k}}\left(C_{\mathrm{Ex}}\right)}{2 N_{k}+1} \geq G\left(C_{\mathrm{Ex}}\right)
$$

for all $k=1,2, \ldots$ Now we set $\delta=\frac{G\left(C_{\mathrm{Ex}}\right)}{2}$ and apply Szemerédi's Theorem. Thus, for any $m$ there exists some large $N \in\left\{N_{k}\right\}_{k=1}^{\infty}$ such that $I_{N}$ contains an arithmetic progression of length $m$. Denote this progression $\{a+d l\}_{l=0}^{m-1}$. Now note that

$$
\left\|\frac{1}{\sqrt{m}} \sum_{l=0}^{m-1} e^{i(a+d l) \cdot}\right\|_{L^{2}(\mathbb{T})}=1,
$$

while

$$
\left\|\frac{1}{\sqrt{m}} \sum_{l=0}^{m-1} e^{i(a+d l) \cdot}\right\|_{L^{1}(\mathbb{T})} \leq \frac{\log \left(\frac{m}{2}\right)}{\sqrt{m}} .
$$

(This is the usual bound for the Dirichlet kernel.) Applying Theorem 2.4, for any fixed constant $C_{\mathrm{Ex}}$, lines (19) and (20) lead to the contradiction

$$
\begin{aligned}
1 & =\left\|\frac{1}{\sqrt{m}} \sum_{l=0}^{m-1} e^{i(a+d l) \cdot}\right\|_{L^{2}(\mathbb{T})} \\
& \leq C_{\mathrm{Ex}}\left\|\frac{1}{\sqrt{m}} \sum_{l=0}^{m-1} e^{i(a+d l) \cdot}\right\|_{L^{1}(\mathbb{T})} \\
& \leq C_{\mathrm{Ex}} \frac{\log \left(\frac{m}{2}\right)}{\sqrt{m}}
\end{aligned}
$$

when $m$ is large.

Thus, if a bound on the peak of all transmission signals is given, as one increases the number of total tones available, at some size $N$ the proportion of tones allocated to carry information must decrease in order to satisfy the PAPR constraint.

From this theorem we also see that when tone reservation is used, the subsets chosen as information and compensation sets are very important. In particular, the information set should not have any long arithmetic progressions; however, determining subsets with little additive structure is a very challenging problem.

\section{The InFINITE SET CASE}

Our first step en route to proving the infinite-dimensional form of Theorem 2.6 is again to prove an equivalence between the PAPR reduction problem and a norm equivalence. This equivalence holds for arbitrary orthonormal systems, so we state it in that generality. We give the equivalence statement in Theorem 3.1. In Theorem 3.2 we prove that the PAPR problem is not solvable at positive efficiencies for sets of infinite cardinality.

Let $\left\{\psi_{k}\right\}_{k \in \mathbb{Z}}$ be an orthonormal basis for $L^{2}(\mathbb{T})$. Let $K$ be a subset of $\mathbb{Z}$, and define

$$
X:=\left\{f \in L^{1}(\mathbb{T}): f(t)=\sum_{k \in K} a_{k} \psi_{k}(t)\right\} .
$$

Given a function $s \in X$, we are interested in finding a compensation function $g, g(t)=\sum_{k \in K^{c}} b_{k} \psi_{k}$, such that 
$\|s+g\|_{\infty} \leq C_{\mathrm{Ex}}\|s\|_{2}$. Here we may view the non-linear operator as a map from $L^{2}(\mathbb{T})$ to $L^{2}(\mathbb{T})$, so that $E_{K} s=s+g$. If a map exists so that such a $g$ can be found for every $s \in X$, then we say that the PAPR reduction problem is solvable for the pair $K$ and $\left\{\psi_{k}\right\}_{k \in \mathbb{Z}}$ with extension norm $C_{\mathrm{Ex}}$.

Theorem 3.1: The PAPR problem is solvable for the pair $K$ and $\left\{\psi_{k}\right\}_{k \in \mathbb{Z}}$ with extension norm $C_{\mathrm{Ex}}$ if and only if

$$
\|f\|_{L^{2}(\mathbb{T})} \leq C_{\mathrm{Ex}}\|f\|_{L^{1}(\mathbb{T})}
$$

for all $f \in X$.

Note that, in constrast to the finite set case of Theorem 2.4, here we have a necessary and sufficient condition for solvability. A necessary and sufficient condition will also be given for the discrete finite set case, which is given in Theorem 4.3.

Proof: i.) Assume that the PAPR problem is solvable. Then for all $s(t)=\sum_{k \in K} a_{k} \psi_{k}(t),\|a\|_{l^{2}(\mathbb{Z})} \leq 1$,

$$
\left\|E_{K} s\right\|_{L^{\infty}(\mathbb{T})} \leq C_{\mathrm{Ex}}\|s\|_{L^{2}(\mathbb{T})} \leq C_{\mathrm{Ex}} .
$$

Since $L^{\infty}(\mathbb{T}) \subset L^{2}(\mathbb{T})$,

$$
E_{K} s=\sum_{k \in K} a_{k} \psi_{k}+\sum_{k \in \mathbb{Z} \backslash K} b_{k} \psi_{k} .
$$

Let $f \in X, f(t)=\sum_{k \in K} c_{k} \psi_{k}(t)$, be arbitrary. Then

$$
\begin{aligned}
\left|\sum_{k \in K} a_{k} \bar{c}_{k}\right| & =\left|\sum_{k \in K} a_{k} \bar{c}_{k}+\sum_{k \in \mathbb{Z} \backslash K} b_{k} \bar{c}_{k}\right| \\
& =\left|\frac{1}{2 \pi} \int_{\mathbb{T}} f(t) \overline{E_{K} s(t)} d t\right| \\
& \leq\|f\|_{L^{1}(\mathbb{T})}\left\|E_{K} s\right\|_{L^{\infty}(\mathbb{T})} \\
& \leq C_{\mathrm{Ex}}\|f\|_{L^{1}(\mathbb{T})} .
\end{aligned}
$$

Set

$$
a_{k}=\left\{\begin{array}{cc}
\frac{c_{k}}{\|c\|_{l^{2}}} & c_{k} \neq 0 \\
0 & c_{k}=0
\end{array} .\right.
$$

Then $\left|\sum_{k \in K} a_{k} \bar{c}_{k}\right|=\|c\|_{l^{2}}=\|f\|_{L^{2}(\mathbb{T})} \leq C_{\mathrm{Ex}}\|f\|_{L^{1}(\mathbb{T})}$.

ii.) Assume $\|f\|_{L^{2}(\mathbb{T})} \leq C_{\mathrm{Ex}}\|f\|_{L^{1}(\mathbb{T})}$ for all $f \in X$. Let $a \in l^{2}$ be a sequence, supported in $K$, with only finitely many nonzero terms satisfying $\|a\|_{l^{2}} \leq 1$. Set $s(t)=$ $\sum_{k \in K} a_{k} \psi_{k}(t)$. For $f \in X, f(t)=\sum_{k \in K} c_{k} \psi_{k}(t)$, define the functional $\Psi_{a}$ by

$$
\Psi_{a} f=\sum_{k \in K} a_{k} \bar{c}_{k}
$$

Since

$$
\left|\Psi_{a} f\right| \leq\|a\|_{l^{2}}\|c\|_{l^{2}} \leq\|f\|_{L^{2}(\mathbb{T})} \leq C_{\mathrm{Ex}}\|f\|_{L^{1}(\mathbb{T})},
$$

$\Psi_{a}$ is continuous on $X$. Since $X$ is a closed subspace of $L^{1}(\mathbb{T})$, by the Hahn-Banach Theorem [5], the functional $\Psi_{a}$ has the extension $\Psi_{E}$ to all of $L^{1}(\mathbb{T})$, where $\left\|\Psi_{a}\right\|=\left\|\Psi_{E}\right\|$. The dual of $L^{1}(\mathbb{T})$ is $L^{\infty}(\mathbb{T})$. Thus, for some $r \in L^{\infty}(\mathbb{T})$,

$$
\Psi_{E} f=\langle f, r\rangle
$$

for all $f \in L^{1}(\mathbb{T})$, so that $\left\|\Psi_{E}\right\|=\|r\|_{L^{\infty}(\mathbb{T})}$. Since $L^{\infty}(\mathbb{T}) \subset$ $L^{2}(\mathbb{T}), r$ possesses the unique expansion

$$
r(t)=\sum_{k \in \mathbb{Z}} d_{k} \psi_{k}(t) .
$$

The sequences $d$ and $a$ agree on $K$, and we define $E_{K} s:=r$.

Theorem 3.2: For $K \subset \mathbb{Z}$, let $S(N)=K \cap$ $\{-N, \ldots, 0, \ldots, N\}$. If $\lim \sup _{N \rightarrow \infty} \frac{|S(N)|}{2 N+1}>0$, then the PAPR problem is not solvable for $K$ and the Fourier basis $\left\{e^{i k \cdot}\right\}_{k \in \mathbb{Z}}$.

In particular, this theorem states that if the ratio of the number of basis functions used for transmission to the total number of basis functions does not tend to zero, then arbitrarily high peeks can be constructed that can not be dampened by any compensation function.

Similar questions concerning the sizes of subsets of orthonormal bases that have a norm equivalance have been studied. In [6], Bourgain addresses an $L^{2}-L^{p}$ norm equivalence for $p>2$. The general technique used here to determine a norm equivalence is well-known in the functional analysis and local Banach space community.

Proof: First suppose that the PAPR problem is solvable for $K$ and $\left\{e^{i k \cdot}\right\}_{k \in \mathbb{Z}}$. We develop a contradiction to the equivalence given in Theorem 3.1. Suppose that arbitrary subsets $S(N)$ of $\{-N, \ldots, N\}$ are chosen such that

$$
\limsup _{N \rightarrow \infty} \frac{|S(N)|}{2 N+1}=\delta>0
$$

For any positive integer $k$, using Szemerédi's theorem (Theorem 2.8) again, there exists a large integer $N$ such that $S(N)$ has an arithmetic progression of length $k$. Denote the arithmetic progression $\{a+d l\}_{l=0}^{k-1}$. We again have

$$
\left\|\frac{1}{\sqrt{k}} \sum_{l=0}^{k-1} e^{i(a+d l) \cdot}\right\|_{L^{2}}=1,
$$

while

$$
\left\|\frac{1}{\sqrt{k}} \sum_{l=0}^{k-1} e^{i(a+d l) \cdot}\right\|_{L^{1}} \leq \frac{\log \left(\frac{k}{2}\right)}{\sqrt{k}} .
$$

Applying Theorem 3.1, for any fixed constant $C_{\mathrm{Ex}}$, for $k$ large enough lines (31) and (32) give the contradiction

$$
\begin{aligned}
1 & =\left\|\frac{1}{\sqrt{k}} \sum_{l=0}^{k-1} e^{i(a+d l) \cdot}\right\|_{L^{2}} \\
& \leq C_{\mathrm{Ex}}\left\|\frac{1}{\sqrt{k}} \sum_{l=0}^{k-1} e^{i(a+d l) \cdot}\right\|_{L^{1}} \\
& \leq C_{\mathrm{Ex}} \frac{\log \left(\frac{k}{2}\right)}{\sqrt{k}} .
\end{aligned}
$$

We point out that the sequence of coefficients used to give the contradiction is not at all exotic-it is just a sequence of 1 's placed at the right locations.

If we work with a finite total number of tones and have an extension constant $C_{\mathrm{Ex}}$, then since the constant $C_{\mathrm{Ex}}$ in both aspects of Theorem 3.1 is the same, we can deduce a bound on the longest arithmetic progression in $I_{N}$. Namely, denoting by $k$ the length of the longest arithmetic progression, we have $1 \leq C_{E x} \frac{\log (k)}{\sqrt{k}}$. 
To emphasize the role of the density, we contrast Theorem 3.2 with the following theorem.

Theorem 3.3: (Theorem 7 in [7]) Let $\lambda>1$ be a real number and assume that the subset $K=\left\{n_{k}\right\}_{k=1}^{\infty} \subset \mathbb{Z}$ has the property $\left|n_{k+1}\right| \geq \lambda\left|n_{k}\right|$ for all $k \leq 1$. Then there exists a constant $C_{K}$ such that for all $a \in l^{2}$ supported on $K$ there exists a continuous function $g \in L^{2}(\mathbb{T})$ satisfying $\|g\|_{L^{\infty}} \leq C_{K}\|a\|_{l^{2}}$ with Fourier coefficients satisfying $\hat{g}_{h_{k}}=c_{n_{k}}$. That is, a compensation signal exists.

In the case addressed in Theorem 3.3, a compensation signal can always be found. But, of course, the difference is that the density of $K$ is zero: for every $k$ elements of $K$ we have roughly $\lambda^{k}$ elements in the complement.

\section{THE Discrete CASE}

It is interesting to consider the analogous discrete case for several reasons. First of all, the problem considered is applicable to a large number of areas and is valuable in its own right. The discrete case is important for the PAPR problem because much of the work done with signals is, of course, done with discretized versions of the signals. For example, oversampling and zero-padding are used in the papers [8], [3]. Lastly, in some settings it is possible, using sampling results, to relate discrete properties to analog properties, and therefore it is valuable to understand the behavior in the discrete setting.

Definition 4.1: The $N \times N$ inverse discrete Fourier transform (DFT) matrix is given by

$$
F_{j k}=\frac{1}{\sqrt{N}} e^{-2 \pi i(j-1)(k-1) / N} .
$$

This matrix is denoted $F$, and for $x \in l_{N}^{2}, F x$ denotes this matrix applied to $x$.

Definition 4.2: $l_{N}^{p}$ denotes $\mathbb{C}^{N}$ viewed as a linear space with norm $\|x\|_{l_{N}^{p}}=\left(\sum_{i=1}^{N}\left|x_{i}\right|^{p}\right)^{1 / p}$. The unit ball in $l_{N}^{p}$ is denoted $B_{N}^{p}$, i.e.

$$
B_{N}^{p}=\left\{x \in l_{N}^{p}:\|x\|_{l_{N}^{p}} \leq 1\right\} .
$$

Let $\left\{N_{k}\right\}_{k=1}^{\infty}$ be a subsequence of $\mathbb{N}$, and for each $N_{k}$ let $I_{N_{k}}$ be a subset of $\left\{1, \ldots, N_{k}\right\} . I_{N_{k}}^{c}$ denotes $\left\{1, \ldots, N_{k}\right\} \backslash I_{N_{k}}$. We say the the discrete PAPR problem is solvable for the sequences $\left\{N_{k}\right\}_{k=1}^{\infty}$ and $\left\{I_{N_{k}}\right\}_{k=1}^{\infty}$ if there exists a constant $C_{\text {Ex }}$, such that for each $k$, for all $x \in l_{N_{k}}^{2}$ with $\operatorname{supp}(x) \subset I_{N_{k}}$ there exists a compensation vector $r \in l_{N_{k}}^{2}$ supported in $I_{N_{k}}^{c}$ such that

$$
\|F(x+r)\|_{l_{N_{k}}^{\infty}} \leq \frac{C_{\mathrm{Ex}}}{\sqrt{N_{k}}}\|x\|_{l_{N_{k}}^{2}} .
$$

Theorem 4.3: Let $\left\{N_{k}\right\}_{k=1}^{\infty}$ be a subsequence of $\mathbb{N}$, and let $I_{N_{k}}$ be a subset of $\left\{1, \ldots, N_{k}\right\}$. Let $Y_{k}=\left\{y \in l_{N_{k}}^{2}\right.$ : $\left.\operatorname{supp}\left(F^{*} y\right) \subset I_{N_{k}}\right\}$. The discrete PAPR problem is solvable for the sequence of sets $\left\{I_{N_{k}}\right\}_{k=1}^{\infty}$ with constant $C_{\mathrm{Ex}}$ if and only if

$$
\|y\|_{l_{N_{k}}^{2}} \leq \frac{C_{\mathrm{Ex}}}{\sqrt{N_{k}}}\|y\|_{l_{N_{k}}^{1}}
$$

for all $y \in Y_{k}$.
In general one has $\|y\|_{l_{N_{k}}^{2}} \leq\|y\|_{l_{N_{k}}^{1}}$ for any vector $y$. Here, though, as $k$ increases, we eventually have $C_{\mathrm{Ex}} / \sqrt{N_{k}}<1$, and so the important point is that $C$ remains fixed.

While the proof is very similar to the proof of Theorem 3.2, we include it so that both the analog and discrete cases are presented clearly. We note as well that here, just as in Theorem 3.2 we have both a necessary and a sufficient condition for solvability.

Proof: i.) Assume that PAPR is solvable. Let $N$ be an element of $\left\{N_{k}\right\}_{k=1}^{\infty}$. For $I_{N} \subset\{1, \ldots, N\}$, let $I_{N}^{c}=$ $\{1, \ldots, N\} \backslash I_{N}$. Then for any $x \in \mathbb{C}^{N}$ with $\operatorname{supp}(x) \subset I_{N}$, we can find an extension $r \in \mathbb{C}^{N}$ with $\operatorname{supp}(r) \subset I_{N}^{c}$, such that

$$
F(x+r) \in \frac{C}{\sqrt{N}} B_{N}^{\infty} .
$$

We denote by $l_{N}^{p}\left(I_{N}\right)$ elements of $l_{N}^{p}$ with support contained in $I_{N}$. Denote by $E_{I_{N}}$ the operator that maps $x$ to the compensated vector $x+r$. Then

$$
\left\|F E_{I_{N}} x\right\|_{l_{N}^{\infty}} \leq \frac{C_{\mathrm{Ex}}}{\sqrt{N}}\|x\|_{l_{N}^{2}},
$$

and so $\left\|F E_{I_{N}}\right\|_{l_{N}^{2}\left(I_{N}\right) \rightarrow l_{N}^{\infty}} \leq \frac{C_{\mathrm{Ex}}}{\sqrt{N}}$. As in the analog case, we take a vector $b$ with $\operatorname{supp}(b) \subset I_{N}$, and observe

$$
\begin{aligned}
\left|\left\langle b, E_{I_{N}} x\right\rangle\right| & =\left|\left\langle F b, F E_{I_{N}} x\right\rangle\right| \\
& \leq\|F b\|_{l_{N}^{1}}\left\|F E_{I_{N}} x\right\|_{l_{N}^{\infty}} \\
& \leq\|F b\|_{l_{N}^{1}} \frac{C_{\mathrm{Ex}}}{\sqrt{N}}\|x\|_{l_{N}^{2}} .
\end{aligned}
$$

By setting

$$
x_{k}=\left\{\begin{array}{cc}
\frac{b_{k}}{\|b\|_{l_{N}^{2}}} & b_{k} \neq 0 \\
0 & b_{k}=0
\end{array},\right.
$$

we obtain

$$
\begin{aligned}
\|b\|_{l_{N}^{2}} & =|\langle b, b\rangle|^{2} \\
& =\left|\left\langle b, E_{I_{N}} x\right\rangle\right| \\
& \leq \frac{C_{\mathrm{Ex}}}{\sqrt{N}}\|F b\|_{l_{N}^{1}} .
\end{aligned}
$$

ii.) Let $N$ be an element of $\left\{N_{k}\right\}_{k=1}^{\infty}$. We take an element $c \in l_{N}^{2}$ with $\operatorname{supp}(c) \subset I_{N}$. Let $\Psi_{c}$ be the functional acting on $Y$ by

$$
\Psi_{c} y=\left\langle c, F^{*} y\right\rangle \text {. }
$$

We then have $\left|\Psi_{c} y\right| \leq\|c\|_{l_{N}^{2}}\|y\|_{l_{N}^{2}} \leq \frac{C_{\mathrm{Ex}}}{\sqrt{N}}\|y\|_{l_{N}^{1}}\|c\|_{l_{N}^{2}}$, so that

$$
\left\|\Psi_{c}\right\| \leq \frac{C_{\mathrm{Ex}}}{\sqrt{N}}\|c\|_{l_{N}^{2}} .
$$

Since $Y$ is a closed subspace of $l_{N}^{1}$, by the Hahn-Banach Theorem there exists an extension $\Psi_{E}$ of $\Psi_{c}$ to all of $l_{N}^{1}$ such that $\left\|\Psi_{c}\right\|=\left\|\Psi_{E}\right\| . \Psi_{E}$ can be represented by a vector $r$ so that

$$
\Psi_{E} y=\langle r, y\rangle
$$

for all $y \in l_{N}^{1}$. Let $\bar{c}=F r$. If $y \in Y$ and $y=F x$, then $\langle r, y\rangle=\langle\bar{c}, x\rangle$. Comparing this with equation (40), we see 
that $c$ and $\bar{c}$ must agree on $I_{N}$. That is, $\bar{c}$ is an extension of c. Lastly, using equation (41),

$$
\begin{aligned}
\left\|\Psi_{E}\right\| & =\|r\|_{\infty} \\
& =\|F \bar{c}\|_{\infty} \\
& \leq \frac{C_{\mathrm{Ex}}}{\sqrt{N}}\|c\|_{l_{N}^{2}} .
\end{aligned}
$$

Theorem 4.4: Let $\left\{N_{k}\right\}_{k=1}^{\infty}$ be a subsequence of $\mathbb{N}$ and let $I_{N_{k}}$ be the corresponding sets as defined earlier. If

$$
\limsup _{n \rightarrow \infty} \frac{\left|I_{N_{k}}\right|}{N_{k}}>0,
$$

then the discrete PAPR problem is not solvable.

Proof: As in the proof of Theorem 3.2, there exists an integer $N$ included in the subsequence of $\mathbb{N}$ that contains an arithmetic progression of length $k$. Assume that this progression is $\{a+b l\}_{l=0}^{k-1}$. Let $D$ denote the vector of length $N$ with the value $\frac{1}{\sqrt{k}} e^{2 \pi i(a+b l) t}$ at the entries of the arithmetic progression, where $t$ will be addressed shortly. Then

$$
\begin{aligned}
\|F D\|_{l_{N}^{1}} & =\sum_{j=1}^{N}\left|(F D)_{j}\right| \\
& =\sum_{j=1}^{N}\left|\frac{1}{\sqrt{N}} \sum_{l=1}^{N} e^{-\frac{2 \pi i l j}{N}} D_{l}\right| \\
& =\sum_{j=1}^{N}\left|\frac{1}{\sqrt{N}} \frac{1}{\sqrt{k}} \sum_{l=0}^{k-1} e^{-\frac{2 \pi i(a+b l) j}{N}} e^{2 \pi i(a+b l) t}\right| \\
& =\sum_{j=1}^{N}\left|\frac{1}{\sqrt{N}} \frac{1}{\sqrt{k}} \sum_{l=0}^{k-1} e^{-\frac{2 \pi i b l j}{N}} e^{2 \pi i b l t}\right| \\
& =\frac{1}{\sqrt{k}} \frac{1}{\sqrt{N}} \sum_{j=1}^{N}\left|\sum_{l=0}^{k-1} e^{2 \pi i b l\left(t-\frac{j}{N}\right)}\right| .
\end{aligned}
$$

This calculation holds for any $t$, so we may take the $t$ that minimizes the absolute value:

$$
\begin{aligned}
\min _{t \in[0,1]} \sum_{j=1}^{N}\left|\sum_{l=0}^{k-1} e^{2 \pi i b l\left(t-\frac{j}{N}\right)}\right| & =\min _{t \in[0,1]} \sum_{j=1}^{N}\left|\frac{\sin \pi b k\left(t-\frac{j}{N}\right)}{\sin \pi b\left(t-\frac{j}{N}\right)}\right| \\
& \leq \int_{0}^{1} \sum_{j=1}^{N}\left|\frac{\sin \pi b k\left(t-\frac{j}{N}\right)}{\sin \pi b\left(t-\frac{j}{N}\right)}\right| d t \\
& =\int_{0}^{1} \sum_{j=1}^{N}\left|\frac{\sin \pi b k t}{\sin \pi b t}\right| d t \\
& =N \int_{0}^{1}\left|\frac{\sin \frac{\pi b k t}{N}}{\sin \frac{\pi b t}{N}}\right| d t \\
& =N \frac{1}{b} \int_{0}^{b}\left|\frac{\sin \frac{\pi k t}{N}}{\sin \frac{\pi t}{N}}\right| d t \\
& =N \int_{0}^{1}\left|\frac{\sin \frac{\pi k t}{N}}{\sin \frac{\pi t}{N}}\right| d t \\
& \leq N \log k,
\end{aligned}
$$

where in lines (45), (46) and (47) we use the periodicity and that $b$ is an integer, and where the last step is the bound on the Dirichlet kernel. Now, returning to line (44), and defining $D$ using the $t$ that results in the minimum in the calculation above, we have $\|F D\|_{l_{N}^{1}} \leq \frac{\log k}{\sqrt{k}} \sqrt{N}$. If the discrete PAPR problem is solvable, then by Theorem 4.3, we have a norm equivalence with a factor $C_{\mathrm{Ex}} / \sqrt{N}$. However, we have just shown that $C_{\mathrm{Ex}}$ must be arbitrarily small. This contradiction proves the theorem.

\section{CONCLUSION}

We have addressed the scaling laws of tone reservation as a method to decrease PAPR in OFDM signals. We have shown that when a PAPR constraint is satisfied, then the number of tones used to carry information cannot scale with the total number of tones available. In fact, the ratio of the number of information tones to total tones converges to zero. One may view this result as giving the trade-off between efficiency and PAPR; but, more generally, it shows that there is a trade-off between complexity and PAPR. That is, if a certain strategy yields a bound on PAPR that is independent of the total number of tones, then the strategy must require a greater overhead than tone reservation.

\section{REFERENCES}

[1] S.H. Han and J.H. Lee. An overview of peak-to-average power ratio reduction techniques for multicarrier transmission. Wireless Communications, IEEE, 12(2):56 - 65, April 2005.

[2] J. Tellado and J.M. Cioffi. Efficient algorithms for reducing par in multicarrier systems. In Proc. IEEE ISIT, 1998., page 191, Aug. 1998.

[3] J. Ilic and T. Strohmer. PAPR reduction in OFDM using Kashin's representation. In Signal Processing Advances in Wireless Communications, 2009. SPAWC '09. IEEE 10th Workshop on, pages 444 -448, June 2009.

[4] T. Tao. A quantitative ergodic theory proof of Szemerédi's theorem. Electron. J. Combin., 13(1):Research Paper 99, 2006.

[5] W. Rudin. Real and Complex Analysis. Third Addition. McGraw-Hill International Editions, New York, 1987.

[6] J. Bourgain. Bounded orthogonal systems and the $\Lambda$ (p)-set problem. Acta Math., 162(3-4):227-245, 1989.

[7] H. Boche and V. Pohl. Signal Representation and Approximation Fundamental Limits. European Trans. Telecommun. (ETT), 18(5):445456, Aug. 2007.

[8] C. Tellambura. Computation of the continuous-time PAR of an OFDM signal with BPSK subcarriers. Communications Letters, IEEE, 5(5):185 -187, May 2001. 